\title{
Boetius, Jan; Frederik Boetius; Joachim Kölschbach: Handbuch der versicherungstechnischen Rückstellungen. Bilanzrecht der Versicherungsunternehmen nach nationalem Recht, Solvency II und IFRS
}

\author{
München, C. H. Beck 2021, XLVII und 582 Seiten
}

\section{Heinrich R. Schradin}

Angenommen: 10. November 2021 / Online publiziert: 29. November 2021

(C) Der/die Autor(en) 2021

\section{I.}

Mit dem Abschluss eines privatwirtschaftlichen Versicherungsvertrages verpflichtet sich der Versicherungsnehmer, die vereinbarte Prämie zu leisten und der Versicherer ist verpflichtet, bei Eintritt des Versicherungsfalles, die versprochene Leistung zu erbringen ( $1 \mathrm{VVG})$. Aus technischer Sicht gilt es demnach zunächst, den für die Sicherstellung des Schutzversprechens ausreichenden Kapitalbedarf zu ermitteln. Dies ist zentraler Gegenstand der versicherungsmathematischen Funktion und Kompetenz des Versicherers. Damit aber dieser Kapitalbedarf auch tatsächlich gedeckt werden kann, bedarf es der entsprechenden Kapitalbildung. Es ist mithin die klassische Aufgabe und Kompetenz des Finanzbereichs im Versicherungsunternehmen sicherzustellen, dass die erforderlichen Mittel nicht aus dem Unternehmen abfließen und als gebundenes Kapital verfügbar sind. Dies wiederum erfolgt wesentlich durch die Bildung „,versicherungstechnischer Rückstellungen“.

Im Vergleich zur ersten Auflage aus dem Jahre 1996, die im Untertitel das Handels- und Steuerrecht der Versicherungsunternehmen betont ${ }^{1}$, wird in der soeben erschienenen zweiten Auflage das Bilanzrecht der Versicherungsunternehmen nach nationalem Recht, Solvency II und IFRS hervorgehoben. Damit wird von vornherein klar, dass das Werk das Ziel verfolgt, die jüngsten und gegenwärtigen Auseinandersetzungen um Ansatz und Bewertung versicherungstechnischer Rückstellungen aus unterschiedlichen Perspektiven nachzuvollziehen und gegenüberzustellen. Neben vertieften Betrachtungen der nationalen bilanz- und aufsichtsrechtlichen Rege-

\footnotetext{
1 Boetius, Jan: Handbuch der versicherungstechnischen Rückstellungen. Handels- und Steuerbilanzrecht der Versicherungsunternehmen. Köln, O. Schmidt 1996, XLII und 508 S.
}

Heinrich R. Schradin $(\bowtie)$

Universität zu Köln, Kerpener Str. 30, 50937 Köln, Deutschland

E-Mail: heinrich.schradin@uni-koeln.de 
lungen sollen versicherungstechnisch bzw. aktuariell geprägte Argumentationslinien aufgezeigt und diese wiederum mit Bezug auf die Genesis des internationalen Rechtsrahmens interpretiert werden. Mit dieser übergreifenden, systematischen und zugleich differenzierten Betrachtung versicherungstechnischer Rückstellungen leistet das Werk einen wichtigen Beitrag für Theorie, Studium und Praxis.

Das solchermaßen anspruchsvolle Vorhaben begründet wohl auch, dass sich der Kreis der Autoren um Jan Boetius (Vorsitzender des Vorstands der DKV Deutsche Krankenversicherung AG i.R.) mit Frederik Boetius (Partner KPMG) und Joachim Kölschbach (Partner KPMG) erweitert hat.

Die Rezension einer derart umfassenden Arbeit beschränkt sich naturgemäß auf die Beleuchtung ausgewählter und vermeintlich wesentlicher Aspekte. Auf eine tiefergehende Auseinandersetzung mit Einzelfragen muss daher verzichtet werden. Im Vordergrund steht die Erläuterung der von den Verfassern entwickelten Systematik und Schwerpunktsetzung.

\section{II.}

Ein erster Blick auf die Inhaltsübersicht lässt demzufolge die Strukturänderung gegenüber der ersten Auflage klar erkennen. Das Handbuch ist nunmehr in drei Teile gegliedert. Im ersten Teil werden „Allgemeine Grundsätze“ (S. 1-60) behandelt, der zweite Teil „Rechtsgrundlagen“" ist in die beiden Kapitel „Nationales Recht“" (S. 61-168) sowie „Internationales Recht“ (S. 169-326) gegliedert und der dritte Teil betrachtet „Einzelne versicherungstechnische Rückstellungen (nach nationalem Recht)“ auf den S. 327-562.

Zur erleichterten Handhabung enthält das Handbuch an zahlreichen Stellen die einschlägigen Rechtsnormen im jeweiligen Richtlinien-, Gesetzes- oder Verordnungswortlaut. Dem Textteil sind ein Inhaltsverzeichnis (IX), ein Abkürzungsverzeichnis (XXXIII) sowie das Literaturverzeichnis (XLV) vorangestellt; das Handbuch schließt mit einem umfangreichen Sachregister (S. 563-582).

A. Im ersten Teil befassen sich die Autoren zunächst in $\$ 1$ grundlegend mit der „Bedeutung der versicherungstechnischen Rückstellungen“ (S. 1-40). Der Schwerpunkt der Ausführungen behandelt betriebswirtschaftliche und versicherungstechnische Grundlagen des Versicherungsgeschäfts. Darüber hinaus wird hier auch der Zusammenhang mit der Vermögensanlagetätigkeit der Versicherungsunternehmen hergestellt. Diese allgemeinen Ausführungen zu den wirtschaftlichen und technischen Grundlagen der Versicherung erscheinen besonders wertvoll. Sie ermöglichen dem Leser einen reflektierten Zugang zu den darauffolgenden detaillierten Erläuterungen nationaler und internationaler Bilanz- und Aufsichtsrechtsnormen und den daraus abgeleiteten, jeweils spezifischen Zwecken dienenden Modellen der Versicherung. In diesem Sinne können auch die weiteren in § 1 vorgenommen Einordnungen (persönlicher und sachlicher Geltungsbereich) und Abgrenzungen (Pensionsfonds, betriebliche Altersvorsorge, Sozialversicherung) verstanden werden. 
§ 2 gibt einen Überblick über die maßgeblichen Rechtsgebiete und deren Beziehungen zueinander (S. 40-54). Nationales und internationales Handelsrecht werden hier ebenso adressiert wie das Verhältnis der Handelsbilanz nach HGB zur Steuerbilanz und zur Solvabilitätsbilanz. Schließlich erfolgt eine knappe Kontrastierung der internationalen Rechnungslegung und der Solvabilitätsbilanz. Vor dem Hintergrund der zuvor beschriebenen betriebswirtschaftlichen und technischen Grundlagen wird nun mit Blick auf die unterschiedlichen Zwecksetzungen nationaler und internationaler Rechnungslegung, Gläubigerschutz bzw. Ausschüttungsbemessung einerseits und Kapitalmarkt- bzw. Investoreninformation andererseits, systematisch und gut nachvollziehbar die Verschiedenheit der Rechnungslegungssysteme dargelegt. Dies gilt in gleicher Weise für die Darstellung prinzipieller Bewertungsdivergenzen zwischen Handelsbilanz (Vorsichtsprinzip) und Solvabilitätsbilanz (Marktkonsistenz).

Die grundsätzlichen Ausführungen im ersten Teil enden der in $\S \mathbf{3}$ übergeordnete Bilanzierungsgrundsätze nach nationalem Recht schlaglichtartig beleuchtet (S. 54-60).

B. Teil 2 des Handbuches umfasst rund 260 Textseiten und ist der Darstellung der Rechtsgrundlagen gewidmet. Sehr detailliert werden im ersten Kapitel die zeitliche und inhaltliche Entwicklung nationaler Rechtsgrundlagen aufgezeigt. Unter weitgehender Beibehaltung der Struktur aus der ersten Auflage, werden hier die für das Verständnis der Bildung versicherungstechnischer Rückstellung entscheidenden Normentwicklungen der letzten 25 Jahre auf den Gebieten des Handelsund Körperschaftssteuerrechts sowie der Versicherungsaufsichtsrechts, nachgezeichnet, erläutert und interpretiert.

§ 4 Handelsrecht (S. 61-100), greift in seinem Abschnitt „A. Rechtsentwicklung" auf die Aktienrechtsreform aus dem Jahre 1965 zurück und berücksichtigt Entwicklungslinien u. a. über das Bilanzrichtlinien-Gesetz (1985), Versicherungsbilanzrichtlinie-Gesetz und (1994) das Bilanzrechtsmodernisierungsgesetz (2009). Der darauffolgende Abschnitt „B. EG-Versicherungsbilanzrichtlinie RL 91/674 EWG“ zeichnet ein umfassendes Bild von den auf europäischer Ebene erarbeiteten Regelungen zur handelsrechtlichen Bewertung versicherungstechnischer Rückstellung, deren Auswirkungen durch die Übernahme in das nationale Handelsrecht bis heute gut sichtbar geblieben sind. In diesem Sinne folgerichtig trägt der folgende Abschnitt die Bezeichnung „C. Handelsgesetzbuch“. Inhaltlich werden hier die ergänzenden Vorschriften für die Handelsbücher der Versicherungsunternehmen und Pensionsfonds aus dem 3. Buch des HGB dargestellt und kommentiert. Neben kurzen Erläuterungen der in den $\S \S 341 \mathrm{e}-341 \mathrm{~h}$ explizit genannten Rückstellungen werden Bewertungsgrundsätze und Fragen zur Diskontierung vor und nach dem BilMoG erörtert.

Die Darstellung der nationalen Rechtsentwicklung wird im $§ 5$ mit Blick auf das Körperschaftssteuerrecht fortgesetzt (101-146). Die inhaltlichen Betrachtungen beginnen mit der Körperschaftssteuerreform aus dem Jahre 1977 und beziehen sich auf unterschiedliche Erscheinungsformen versicherungstechnischer Rückstellungen (insb. Rückstellung für Beitragsrückerstattung, Deckungsrückstellung, Schadenrückstellung, Drohverlustrückstellung, Schwankungsrückstellung) und den Ein- 
flüssen der im Zeitverlauf erfolgten Steuerrechtsänderungen. Auch hier erfolgt eine Kommentierung der Regelungen zur realitätsnäheren Bewertung und zur Abzinsung.

§ 6 Versicherungsaufsichtsrecht (S. 146-168) ist zunächst in Abschnitt A mit der Darstellung und Kommentierung der Rechtsentwicklung befasst, ausgehend von der Aktienrechtsreform 1965 über die Dritte EG-Richtliniengeneration 1992, die Richtlinien zu Solvabilität II 2009 und Omnibus II 2014 bis hin zum Finanzaufsicht-Modernisierungsgesetz 2015. Die aufsichtsrechtlichen Auswirkungen für den Ansatz und die Bewertung versicherungstechnischer Rückstellungen wesentlichen Grundsätze und Anwendungen werden im Abschnitt B Versicherungsaufsichtsgesetz erläutert. Die vergleichsweise knappen Ausführungen zu den einschlägigen $\S \S$ des VAG erscheinen insb. unter didaktischen Aspekten wohlbegründet. Die Autoren vermeiden auf diese Weise mögliche Redundanzen, die sich mit Blick auf das nachfolgende Kapitel 7 Solvabilität II, leicht hätten ergeben können.

Das zweite Kapitel der Rechtsgrundlagen ist mit Internationales Recht überschrieben und befasst sich in $\S 7$ mit Solvency II und in $\S 8$ mit der Internationalen Rechnungslegung nach IFRS. Beide Kapitel sind in der zweiten Auflage neu hinzugekommen und wurden von den beiden ebenfalls neu hinzugetretenen Autoren, Frederik Boetius (Solvency II) und Joachim Kölschbach (Internationale Rechnungslegung), bearbeitet. Diese Erweiterung der Perspektiven ist nicht einfach nur zeitgemäß, vielmehr erreicht das Handbuch der versicherungstechnischen Rückstellungen damit sowohl unter theoretisch-inhaltlichen und didaktischen Gesichtspunkten als auch mit Blick auf den praktischen Anwendungsbezug ein Niveau, das es in der Literatur so bislang nicht gegeben hat.

Stand der Kommentierung in $§ 7$ Solvency II ist die RL 2009/138/EG vom 13.01.2019 und VO (EU) 2015/vom 30.07.2020. Der Paragraph umfasst $130 \mathrm{~S}$. (S. 169-299) und ermöglicht so, eine angemessene Darstellung von Grundlagen der europäischen Finanzaufsicht von Versicherungsunternehmen, wie sie sich insb. aus der Solvency II-RL aus dem Jahr 2009 ergeben (Aufsichtsziele, Drei-Säulen-Aufsicht, Bilanzkonzeption). Darüber hinaus bleibt genügend Raum für die Erläuterung der für die aufsichtsrechtliche Bewertung versicherungstechnischer Rückstellungen einschlägigen Grundsätze (Marktkonsistenz, Vorsichtsprinzip, Verlässlichkeit und Objektivität). Im Anschluss erfolgt dann die sehr ausführliche und zugleich kritische Diskussion der für die aufsichtsrechtliche Rückstellungsbewertung zentralen Methodik zur Ermittlung des Besten Schätzwertes und der Risikomarge (S. 206-250). Spartenspezifika für die Darstellung von Verpflichtungen aus der Lebensversicherung und der Nicht-Lebensversicherung werden ebenso erläutert, wie Besonderheiten der Abbildung der passiven Rückversicherung. Neben diesen vor allem unter theoretischen Gesichtspunkten interessanten Themen wird auf die unter praktischen Aspekten herausfordernden Themenfelder Datenqualität, Verfahren und Governance ein besonderes Augenmerk gelegt. Desweiteren werden in diesem für das Handbuch zentralen Kapitel besondere Vorschriften für einzelne Versicherungszweige, und Vertragsformen sowie für Versicherungsgruppen beschrieben. Schließlich werden dann auch Übergangsmaßnahmen (Run-Off, Zinstransitional, Rückstellungstransitional) erläutert.

Stand der Kommentierung des IFRS 17 in \$ 8 Internationale Rechnungslegung ist dessen Fassung vom 17. Mai 2017 unter Berücksichtigung der Änderungsvor- 
schläge des IASB vom Juni 2019. Dieser Paragraph ist zwar vergleichsweise kürzer gehalten (S. 299-326), erscheint aber mit Blick auf das Handbuch der versicherungstechnischen Rückstellungen durchaus angemessen. Zunächst werden die die internationale Rechnungslegung prägenden allgemeinen Grundsätze einführend und mit Blick auf die Abgrenzung von Versicherungsverträgen und die wirtschaftliche Betrachtungsweise (,substance over form“) i.S. des IFRS 17 dargestellt und erläutert. Im Anschluss folgt eine überblicksartige Erörterung der spezifisch aus dem IFRS 17 abgeleiteten Bewertungsgrundsätze. Ab S. 307 erfolgt schließlich eine sehr systematische und ausführliche Erläuterung und Kommentierung bewertungsrelevanter Gegenstandsbereiche, wie diese im IFRS 17 adressiert werden: Zahlungsstromperspektive, Zeitwert des Geldes und Barwertbetrachtung, Ausweis und Anpassung der versicherungstechnischen Risikotragung, Ansatz und Bewertung der Servicemarge zur Periodisierung des Gewinnausweises, Ausweis der Verpflichtungen aus der Schadenrückstellung und aus Überschussbeteiligung sowie die Abbildung von Forderungen aus der passiven Rückversicherung. In den darauffolgenden Abschnitten (S. 321 ff.) werden die Regelungen des IFRS 17 mit Blick auf die Ergebnisrechnung und die prinzipienorientierten Anhangangaben dargestellt und erläutert.

C. Teil 3 des Handbuches umfasst knapp 240 Textseiten und ist der Darstellung einzelner versicherungstechnischer Rückstellungen nach derzeit geltendem deutschem Recht gewidmet. Grundsätzlich folgt die zweite Auflage des Handbuchs nun strukturell der aus der ersten Auflage bekannten Vorgehensweise. Inhaltliche Anpassungen folgen der Rechtsentwicklung der vergangenen 25 Jahre unter Berücksichtigung einschlägiger Kommentierungen und Veröffentlichungen. Struktur und Inhalt der nachfolgenden Paragrafen folgen dabei konsequent einer, nicht zuletzt steuerrechtliche geprägten, dynamischen Bilanzauffassung und damit der Interpretation der Rückstellungsbildung als Abzugsposten im Rahmen der Ermittlung des Periodenergebnisses (Ausschüttungs- bzw. Steuersperre). Der Einzeldarstellung wesentlicher versicherungstechnischer Rückstellung ist in § 9 ein Überblick vorangestellt, der das ebenfalls aus der ersten Auflage wohlbekannte „ABC versicherungstechnischer Rückstellungen“ enthält und insb. den noch nicht tiefer in der Materie eingedrungenen Lesern eine wertvolle Orientierungshilfe an die Hand gibt (S. 327-337).

Im weiteren Verlauf folgen detaillierte Ausführungen zu 10 versicherungsspezifischen Rückstellungspositionen ( $§ 10-19$ ), die an dieser Stelle lediglich genannt werden:

- $\S 10$ Alterungsrückstellung, S. 338-375,

- $\$ 11$ Beitragsrückerstattung (BE)/Rückstellung für Beitragsrückerstattung (RfB), S. 375-411,

- $\$ 12$ Beitragsüberträge (BÜ), S. 412-425,

- $\$ 13$ Deckungsrückstellung (DR), S. 426-442,

- $\$ 14$ Drohende Verluste aus schwebenden Geschäften/Rückstellung für drohende Verluste (RdV), S. 442-454,

- $\S 15$ Großrisikenrückstellung (GroßrisikenR), S. 454-477,

- §16 Kumulrisikenrückstellung (KumulrisikenR), S. 478-497, 
- § 17 Schadenrückstellung (SR), S. 497-543,

- $\$ 18$ Schwankungsrückstellung (SchwR), S. 543-560,

- $\$ 19$ Stornorückstellung (StornoR), S. 560-562.

Die Beschreibungen und Kommentierungen sind perspektivisch breit angelegt. Durchgängig geht hier eine grundlegende Erörterung versicherungstechnischer Aspekte der systematischen und differenzierten Darstellung und Interpretation handels- und steuerrechtlicher Regelungssachverhalte voraus. Dem professionellen Hintergrund der Autoren bzw. des Autors folgend, verharren die Ausführungen dabei keineswegs auf theoretischem Niveau; zahlreiche praxisnahe Beispiele, Modellrechnungen sowie erfahrungsgestützte Argumente und Hinweise ziehen sich wie ein roter Faden durch die Ausführungen in den einzelnen Paragraphen.

\section{III.}

Die neue Auflage des Handbuchs der versicherungstechnischen Rückstellungen wird den von den Autoren im Vorwort formulierten Ansprüchen in hohem Maße gerecht. Diese grundsätzliche Wertung bezieht sich auf sämtliche Dimensionen und Perspektiven des Werks.

Rein formal betrachtet ist der Umfang der reinen Textseiten von $366 \mathrm{~S}$. in der ersten Auflage auf nunmehr 561 deutlich gestiegen. Die gilt umso mehr, als Schriftgrad und Formatierung in der neuen Auflage den Vorlagen entspricht, die aus den von C.H. Beck verlegten Kommentaren bekannt sind. Allerdings finden sich in der ersten Auflage die einschlägigen Rechtsnormen explizit im 120 S. umfassenden Anhang aufgeführt, wohingegen die relevanten Normtexte nun regelmäßig am Beginn der jeweiligen Paragrafen zu finden sind. Auch die insoweit geänderte Vorgehensweise erscheint geeignet, dem Nutzer den Zugriff auf die unterschiedlichen Rechtsquellen zu erleichtern.

Vergleichbar mit der ersten Auflage handelt es sich um eine eng am geltenden Recht orientierte, in hohem Maße differenzierte Beschreibung und Kommentierung der jeweils einschlägigen Ansatz- und Bewertungsvorschriften für versicherungstechnische Rückstellungen. Wie es der Titel des Werkes verspricht, steht im neuen „Handbuch der versicherungstechnischen Rückstellungen“ der Anwendungsnutzen klar im Vordergrund. Eine breit angelegte Diskussion strittiger Rechtsfragen, wie dies beispielsweise in einer Gesetzeskommentierung erwartet werden kann, ist nicht Gegenstand des Werks ${ }^{2}$. Vielmehr arbeiten die Autoren betriebswirtschaftliche und versicherungstechnische Grundlagen der jeweiligen Rückstellungspositionen gezielt heraus und leisten so, gerade für die anwendungsorientierte Bilanzierungspraxis, einen wertvollen Beitrag für das Verständnis der jeweiligen Abbildungsnorm. Insofern das Handbuch in seiner zweiten Auflage aber über das nationale Handels- und

\footnotetext{
2 Jäger, Bernd: Zur bilanziellen Behandlung versicherungstechnischer Rückstellungen nach Handels- und Steuerrecht. Betriebswirtschaftliche Diskussion und zugleich Stellungnahme zu Boetius, Jan: Handbuch der versicherungstechnischen Rückstellungen. Handels- und Steuerbilanzrecht der Versicherungsunternehmen, in: ZVersWiss, 88. Band, 1999, S. 149-206.
} 
Steuerrecht hinausreicht und nationale aufsichtsrechtliche Regelungen sowie das internationale Bilanzrecht (IFRS) und das europäische Aufsichtsrecht (Solvency II) umfasst, werden die Zusammenhänge zwischen den unterschiedlichen Zwecksetzungen und Bilanzierungsziele einerseits und den jeweiligen Normen offensichtlich. Die vorgestellten Ansätze sind dabei theoretisch vielfältig und inhaltlich anspruchsvoll. Als wäre bereits dies noch nicht genug, dokumentieren die Schwerpunktsetzungen und die inhaltlichen Kommentierungen die langjährige Praxiserfahrung der Autoren.

Aus diesen Gründen eignet sich das Handbuch der versicherungstechnischen Rückstellungen in besonderer Weise nicht nur für theoretisch interessierte Leser, sondern ebenso für anwendungsorientierte Praktiker.

Funding Open Access funding enabled and organized by Projekt DEAL.

Open Access Dieser Artikel wird unter der Creative Commons Namensnennung 4.0 International Lizenz veröffentlicht, welche die Nutzung, Vervielfältigung, Bearbeitung, Verbreitung und Wiedergabe in jeglichem Medium und Format erlaubt, sofern Sie den/die ursprünglichen Autor(en) und die Quelle ordnungsgemäß nennen, einen Link zur Creative Commons Lizenz beifügen und angeben, ob Änderungen vorgenommen wurden.

Die in diesem Artikel enthaltenen Bilder und sonstiges Drittmaterial unterliegen ebenfalls der genannten Creative Commons Lizenz, sofern sich aus der Abbildungslegende nichts anderes ergibt. Sofern das betreffende Material nicht unter der genannten Creative Commons Lizenz steht und die betreffende Handlung nicht nach gesetzlichen Vorschriften erlaubt ist, ist für die oben aufgeführten Weiterverwendungen des Materials die Einwilligung des jeweiligen Rechteinhabers einzuholen.

Weitere Details zur Lizenz entnehmen Sie bitte der Lizenzinformation auf http://creativecommons.org/ licenses/by/4.0/deed.de. 